\title{
Introduction of the GyneFix intra-uterine device into the UK: Client satisfaction survey and casenotes review
}

\author{
Joanne Dennis, MRCGP, MFF, Career Grade Trainee; Anne Webb, MRCGP, MFFP, MRCOG, Consultant; Meera Kishen, MD, DGO, \\ Dip.Ven., MFFP, Consultant, Abacus Centres for Contraception and Reproductive Health, North Mersey Community NHS Trust, \\ Liverpool, $U K$.
}

Correspondence: Dr Jo Dennis, Central Abacus, 40-46 Dale St, Liverpool, L2 5SF. Tel: 01512842500 , Fax: 01512932005.

(Accepted 30 $0^{\text {th }}$ March 2001)

The Journal of Family Planning and Reproductive Health Care 2001: 27(3): 139-144

\begin{abstract}
Objective. To assess the first year's use with the GyneFix intra-uterine device. This has been used in Liverpool since early 1997. It is offered mainly to nulliparous women wishing to use an effective non-hormonal method and parous women who have had pain with, or expulsion of, a framed device previously. It is also used as emergency contraception.

Design. Retrospective study, by means of casenote review and client questionnaire.

Participants. All GyneFix users from February 1997 to January 1998; 215 women in total.

Main outcome measures. Data were extracted from casenotes to determine reasons for choice of GyneFix, parity, whether the insertion was planned or as an emergency measure, problems reported at follow-up and reasons for removal. Users were asked by questionnaire to comment on insertion procedure, menstrual or other changes since insertion and to state their overall satisfaction.
\end{abstract}

Results. Over half of the users (57\%) were nulliparous and a quarter of insertions were for emergency contraceptive use. A third reported that insertion was 'very painful'. Half reported that their periods had become heavier since insertion, but only $8 \%$ said that they were now unmanageable. Unacceptable bleeding was the most common reason for removal. Some reported intermenstrual bleeding and dysmenorrhoea. Satisfaction with the GyneFix was high; $86 \%$ of questionnaire responders said that they would recommend it to a friend.

Conclusion. The GyneFix is well accepted in Liverpool in appropriately selected clients, and is an effective nonhormonal method for nulliparous as well as parous women.

\section{Key message points}

- The GyneFix is an effective alternative to hormonal methods of contraception and is acceptable to nulliparous women

- Users should be at low risk of sexually transmitted infection and consideration should be given to screening for Chlamydia trachomatis prior to insertion.

- Insertion can be painful, especially in nulliparous women.

- Periods may become heavier, although within acceptable limits for most. GyneFix should not be offered to a woman who already suffers heavy periods.

- Most users are satisfied with the GyneFix. Of those that are not, the most common reason is heavy periods.

\section{Introduction}

The GyneFix intra-uterine device (IUD) was introduced into the UK in 1997. It is a frameless copper-bearing device, designed to reduce the incidence of bleeding, pain and expulsion seen with framed devices. It is marketed as being a reliable alternative to hormonal contraception, particularly for nulliparous women in whom there may be incompatibility between the uterine cavity and the frame of a standard device, causing discomfort, bleeding and expulsion. Clinical trials have confirmed that the GyneFix is effective and acceptable to nulliparous as well as parous women, ${ }^{1}$ although to date there has not been a randomised trial involving nulliparous women comparing GyneFix to other copper IUDs.

The Contraception and Reproductive Health Service in Liverpool was the first in the UK to offer this new device and commence the cascade of training to other service providers. In our service the IUD is a popular choice and is also offered routinely as an option for emergency contraception where there are no contraindications, both to nulliparous and parous women. As many requests for emergency contraception are from young, nulliparous women, the availability of an IUD that would be acceptable as an ongoing method to this group was particularly welcomed.

Insertions started in Liverpool in February 1997. The GyneFix IN (interval) is the version used in our service. In order to assess the first years' experience with the device, a retrospective study was carried out. This was by systematic casenote review and postal questionnaire. The purpose of the study was to look at reasons for choosing the GyneFix over a framed device, events at and subsequent to insertion, and to assess client satisfaction.

Introduction of the GyneFix to Liverpool

At the time of introduction, guidelines were drawn up to help clinicians decide who would benefit from using a GyneFix rather than a framed device. Based on information available ${ }^{1-3}$ it was decided the device would be offered to:

- nulliparous women wishing to use an effective nonhormonal method

- parous women who had experienced a previous IUD expulsion

- parous women who had suffered pain with a framed IUD previously

- parous women who, after full counselling on all available methods, preferred to use a GyneFix rather than a framed device.

There was some initial confusion about the effect the GyneFix might be expected to have on menses. Thus some women not wishing to use a hormonal method were offered, or requested, the GyneFix instead of a framed device in the hope that it would not worsen their already-heavy periods.

GyneFix is only offered as emergency contraception where long-term use is intended. If removal at the next 
period is intended, then a less-expensive framed device is used.

As many insertions are in nulliparous women, where dilatation of the cervix may be necessary, local anaesthetic may be needed. An intra-cervical block technique is used in our service. Local anaesthetic is injected into the cervical lip where the tenaculum is to be placed, and then into the cervix at the level of the internal os through the cervical canal at 3 and 9 o'clock.

\section{Chlamydia testing}

At the time of IUD insertion, infection may be introduced into the uterus, resulting in pelvic inflammatory disease (PID). This may lead to tubal damage and subsequent subfertility. It is now known that the risk of PID is largely related to the presence or acquisition of sexually transmitted infection (STI) - usually Chlamydia - and not the IUD or parity of the woman per se. This information is shared with all potential IUD users in our service, enabling each client to make an informed decision as to whether an IUD is the correct method for her. Testing for Chlamydia is carried out if felt necessary by clinician and/or client, for example where the relationship is new or where there are doubts about monogamy. Results are awaited, where possible, prior to insertion. If the insertion is as an emergency postcoital measure, then insertion must proceed without knowledge of the result.

\section{Method}

Casenotes were reviewed to determine reason for choice of GyneFix over a framed device, parity (nulliparous meaning never pregnant or no pregnancy over 24 weeks), whether the insertion was planned or an emergency measure, and problems reported subsequently at follow-up.

A questionnaire designed to give a qualitative assessment of experience with the method was sent to all clients consenting to correspondence at home. Questions related to the insertion procedure, menstrual and other changes since insertion, and to overall satisfaction. Answers were multiple-choice style, with blank spaces for comments. Questionnaires were sent out 2 months after the last of the 215 insertions had taken place. Where no reply was received within 3 weeks, a second questionnaire was sent. Where there was still no reply, a questionnaire was placed in the casenotes so that the woman could be approached opportunistically when she next attended clinic and asked to complete the questionnaire. Women who had declined correspondence home were approached opportunistically in the same way. A short pilot was carried out amongst clinic attenders before finalising the questionnaire, and Ethical Committee approval was sought prior to distribution.

Information was entered onto an Excel spreadsheet. The last systematic casenote review was carried out in April 1999, although the spreadsheet continues to be updated as further information becomes available. Events up to August 2000 are included.

\section{Results}

Over the first year, from February 1997 to January 1998, a total of 215 insertions were carried out. Table 1 shows numbers of subjects according to parity, divided into emergency and planned insertions. Over half of the insertions $(123 ; 57 \%)$ were in nulliparous women, with 92 $(43 \%)$ in parous women. A quarter $(56 ; 26 \%)$ were for
Table 1 Total number of GyneFix insertions from January 1997 February 1998, showing parity and timing of insertion (percentages in brackets)

\begin{tabular}{llll}
\hline & Nulliparous $(\%)$ & Parous $(\%)$ & TOTAL (\%) \\
\hline Emergency & $41(19)$ & $15(7)$ & $56(26)$ \\
Planned & $82(38)$ & $77(36)$ & $159(74)$ \\
TOTAL & $123(57)$ & $92(43)$ & $215(100)$ \\
\hline
\end{tabular}

emergency use. The excess of nulliparous over parous users stems mainly from the emergency users. There was one abandoned insertion, due to inability to advance the uterine sound through the cervical canal. This client was provided with an alternative method of contraception and has not been included in the analysis.

Table 2 Source of follow-up data (percentages in brackets)

\begin{tabular}{lc}
\hline Source of follow up data & Numbers (\%) \\
& $\mathrm{N}=215(100)$ \\
\hline Questionnaire and casenotes & 105 \\
Questionnaire only & 27 \\
Total returning questionnaire & $132(61)$ \\
& \\
Casenotes only & 51 \\
Total for whom follow up information available & $183(85)$ \\
& \\
No follow-up & 32 \\
TOTAL & $215(100)$ \\
\hline
\end{tabular}

One hundred and eighty-nine clients were sent questionnaires (Table 2). The remaining 26, who had not consented to correspondence home, had questionnaires placed in their casenotes, along with those who had consented to correspondence home but failed to return two mailed questionnaires. Completed questionnaires were returned by nearly two-thirds $(132,61 \%)$. There was no significant difference in parity between the responders and non-responders using chi-square testing, with 73 (55\%) of the respondents being nulliparous and $59(45 \%)$ parous.

Thirty-two women (18 nulliparous, 14 parous) have neither returned a questionnaire nor attended clinic since insertion; thus follow-up information is available for 183 $(85 \%)$ out of the total 215 women.

The time interval between GyneFix insertion and completion of the questionnaire ranged from two to 20 months. The time interval between insertion and clinic follow-up ranges from a few days to 39 months.

\section{Reason for choosing GyneFix}

Table 3 shows reasons for the choice of GyneFix over a framed device. Data were extracted from casenotes for all 215 women. Most women chose the GyneFix because of

Table 3 Reason for choosing GyneFix over a framed device, as recorded in casenotes (percentages in brackets)

\begin{tabular}{lc}
\hline Reason for choosing GyneFix & No. of women $(\%)$ \\
& $\mathrm{N}=215(100)$ \\
\hline Nulliparity & $113(53)$ \\
Client preference, no clinical reason & $50(23)$ \\
Previous IUD related dysmenorrhoea & $20(9)$ \\
Previous IUD expulsion & $10(5)$ \\
Previous IUD related bleeding problems & $5(2)$ \\
Dysmenorrhoea currently & $5(2)$ \\
Heavy menses currently & $1(1)$ \\
Unknown reason & $10(5)$ \\
\hline
\end{tabular}


nulliparity or previous IUD related pain or expulsion. Twenty-three percent of insertions were at the client's request, with no obvious clinical reason why the GyneFix might be a better choice than a framed device (all parous women). Five women chose the device because they had dysmenorrhoea, in the hope that the GyneFix would not worsen it. Similar reasons were documented for one woman whose natural menses were heavy and five women who had had excessive menstrual bleeding with a framed IUD.

\section{The insertion}

Clients were asked by questionnaire how painful the insertion was. A third responded 'very painful' and $31 \%$ 'more painful than I expected but bearable' (Table 4). More nulliparous women than parous responded to these options.

Amongst those accepting intracervical block there was no observed reduction in those reporting that insertion was very painful. Seventy-three women had a cervical block. Forty-two of these responded to the questionnaire, 15 stating that insertion had been 'very painful'. Thus $36 \%$ of questionnaire responders having a cervical block reported insertion to be 'very painful'.

Table 4 Questionnaire responder's perception of pain on insertion (percentages in brackets)

\begin{tabular}{llll}
\hline & $\begin{array}{l}\text { Nulliparous (\%) } \\
\mathrm{N}=73(100)\end{array}$ & $\begin{array}{l}\text { Parous (\%) } \\
\mathrm{N}=59(100)\end{array}$ & $\begin{array}{l}\text { Total }(\%) \\
\mathrm{N}=132(100)\end{array}$ \\
\hline $\begin{array}{l}\text { Very painful } \\
\begin{array}{l}\text { More painful than } \\
\text { expected but bearable }\end{array}\end{array}$ & $25(34)$ & $17(29)$ & $42(32)$ \\
$\begin{array}{l}\text { As expected } \\
\text { Less painful than }\end{array}$ & $14(19)$ & $15(25)$ & $41(31)$ \\
$\begin{array}{l}\text { expected } \\
\text { Painless }\end{array}$ & $7(10)$ & $10(19)$ & $25(19)$ \\
\hline
\end{tabular}

\section{Chlamydia testing}

One hundred and fifty-two women (71\%) had a Chlamydia test. Three results were positive, giving a rate of $2 \%$. All three were emergency insertions in nulliparous women under the age of 25. All were referred to the local genitourinary medicine (GUM) clinic. One had developed upper abdominal but no pelvic pain and a presumptive diagnosis of Fitz-Hugh Curtis syndrome was made. The other two were asymptomatic, and all three opted to retain the GyneFix. There were no known cases of PID amongst those in whom Chlamydia testing was not carried out.

\section{Menstrual changes since insertion}

Clients were asked, both at clinic follow-up and by questionnaire, what, if any, problems they had experienced. The results in Table 5 are amalgamated results from both

Table 5 Numbers (percentages in brackets) of clients reporting menstrual changes since insertion of GyneFix, combined casenote and questionnaire data

Nulliparous (\%) Parous (\%) TOTAL (\%)

$\mathrm{N}=103(100) \quad \mathrm{N}=80(100) \quad \mathrm{N}=183(100)$

Periods become

unmanageably heavy

$4(4)$

11 (14)

$15(8)$

Periods heavier but

42 (41)

Inter-menstrual bleeding 20 (19)

$40(50)$

$82(45)$

Pelvic pain/

dysmenorrhoea

$13(13)$

$12(15) \quad 25(14)$

casenote and questionnaire analysis for the 183 women on whom follow-up information is available (103 nulliparous and 80 parous).

Over half reported that their periods had become heavier since insertion. The remainder said that they were the same or lighter. Those reporting that their periods were now intolerably heavy were mostly parous women. One of these women had reported heavy bleeding with a framed IUD. A number of women reported inter-menstrual bleeding and the development of dysmenorrhoea or pelvic pain since insertion. Most of these had also reported heavier periods. There were no contraceptive failures, but one pregnancy resulted from unnoticed expulsion.

\section{Other events}

There have been 48 known removals. Figure 1 shows removals for nulliparous and parous users. A third (16) were due to bleeding problems, either heavy menses (11) or intermenstrual bleeding (5). The remainder were for a variety of reasons, including: to conceive (11 removals); pain (10 removals; two within 30 minutes of insertion due to persistent pain on embedding the anchoring knot in the fundal myometrium, three dysmenorrhoea, four nonmenstrual pelvic pain, one dyspareunia); two cases of suspected PID (swabs negative in both, one subsequently found to have an ovarian cyst), and one pregnancy, although conception was prior to insertion. Eight removals were for unrelated or non-medical reasons, such as no further need for contraception. Using chi-square testing, there is no significant difference in removal rates for bleeding and pain between nulliparous and parous users.

Figure 1 Numbers of and reasons for GyneFix removal

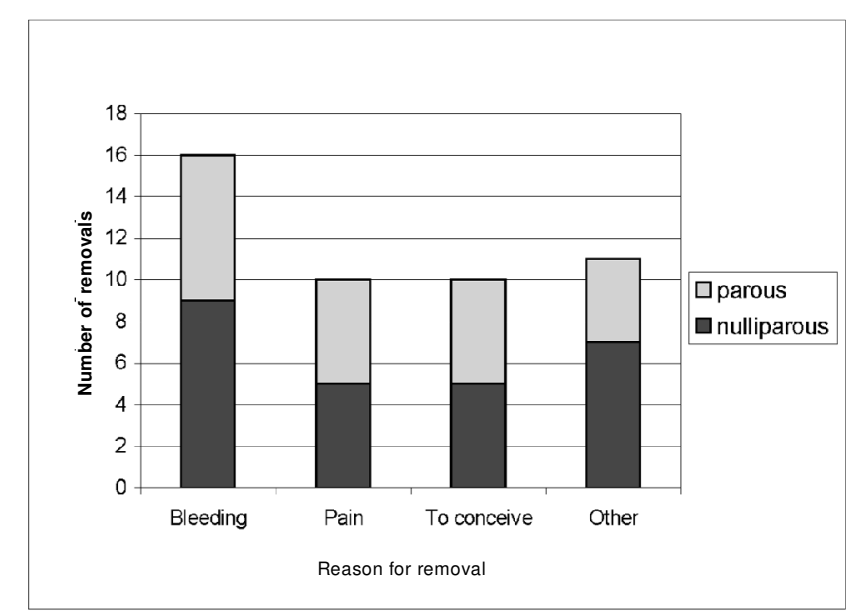

There have been 19 expulsions about which we are aware. Six of these were within 3 months of insertion. Most of the devices expelled were inserted by clinicians undergoing insertion training. One unnoticed expulsion resulted in pregnancy, diagnosed 20 months after insertion. Six clients opted for re-insertion and continued to be included in the survey.

\section{Comments from users}

Clients were asked for their comments on the method. Most of these were encouraging:

'The best method I've ever used'

'It doesn't cause period pains like my old coil did'

'I forget it's there - it's very convenient' 
'The staff were friendly and helpful'

But some negative comments were also received:

'I was disappointed about my heavy periods'

'I wasn't really prepared for just how painful insertion would be'

'I was devastated when it fell out'

\section{Would you recommend the GyneFix to a friend?}

To this all-important question, the majority ( $86 \%)$ answered 'Yes'. Although 13\% said 'No', most of these were because of heavy periods. Slightly more nulliparous than parous users answered 'Yes' to this question ( $88 \%$ Vs $80 \%)$.

\section{Discussion}

The introduction of a new treatment, or in this case method of contraception, is an opportunity to look at both the method itself and clinical standards within the service. If a new service is to become established, then there needs to be some evidence that there is a requirement for that service rather than providing it just because something new is available.

When the GyneFix was first introduced into the service it was felt necessary to draw up some guidelines about who should be offered the device, or more importantly, who might benefit from using the GyneFix rather than a framed device. Nulliparous women and those who had had expulsion of a framed device appeared most likely to benefit, based on published evidence available at the time.

Two randomised comparative trials comparing prototype versions of the GyneFix (identical in all but insertion instrument, which was redesigned as a result of problems with expulsion) to the Copper T380A did not show any advantage of the GyneFix over the framed device in parous women only. 2,3

A large non-comparative study in both nulliparous and parous women reported low removal rates for bleeding and pain and very low expulsion rates in all users. ${ }^{1}$ The authors concluded that the absence of a frame explains the low incidence of side effects and that the GyneFix is effective and well accepted by nulliparous women. As bleeding and pain were not separated in the analysis, it was not possible to discern whether most problems resulting in removal were related to bleeding or pain. Thus some initial insertions were carried out in women reporting either heavy periods currently or heavy periods with a framed device in the past, in the hope that the GyneFix would not worsen the periods to the extent that a framed device might.

As a result of over $50 \%$ reporting that their periods became heavier with the device, it is now standard practice to advise that periods are likely to become heavier. Interestingly though, none of the five women choosing the GyneFix because they had had IUD-induced bleeding problems previously has had her device removed, and only one has reported unmanageably heavy periods. Intermenstrual bleeding occurred in almost a fifth of users most of these had also reported heavier periods.

It was surprising that up to $15 \%$ reported that their periods had become more painful since insertion. Most of this was reported by questionnaire - complaints of dysmenorrhoea at follow-up have been rare. The impression that the clinicians involved with GyneFix have developed is that most women do not suffer IUD-related pain. Some of these women had discontinued the combined pill when the GyneFix was inserted, which may account for a worsening of dysmenorrhoea. Heavier menses may also have contributed.

It is possible that those responding to the questionnaire or attending clinic after a longer time may have had time both for problems to settle and for new ones to emerge. Due to the disparity in lengths of time involved, it is not possible to draw any further conclusions or attempt to apply significance testing. Anecdotally, some women have reported that initially heavy periods tended to settle over the first few months of use.

Since this study, the 3-year interim results of a large randomised trial currently underway in China have been published. ${ }^{4}$ Women are randomised to receive either the GyneFix or the TCu380A. The removal rate for both bleeding and pain is lower for the GyneFix than the framed device users, although the figures do not reach significance. Only parous women have been recruited.

It is as anticipated that more nulliparous women than parous reported that insertion was painful. It is surprising that the use of intra-cervical block did not lead to fewer reporting that insertion was painful. Some had local anaesthetic after the procedure had already begun, due to feeling discomfort on cervical manipulation. Also, cervical anaesthesia will not have any impact on pain felt on penetration of the fundal myometrium. It is not possible to make any further analysis of whether pain was felt on cervical manipulation or myometrial penetration using the data collected, but it shows that local anaesthetic is not always necessary even in nulliparous women. Clinicians in our service have observed that the less nervous the woman, the less likely she is to suffer pain. Pain tends to resolve quickly once the device is in place. Giving oral analgesia prior to the procedure may be of benefit.

Women considered suitable for IUD use should have an inherently low risk of STI. The three women whose tests were positive were all under the age of 25 , consistent with the trend of Chlamydia infection seen in the general population. The positivity rate of $2 \%$ is lower than the reported prevalence in under-25s in the UK (upwards of $5 \%$ nationally; ${ }^{5}$ as high as $13 \%$ in some parts of Liverpool $^{6}$ ). Those undergoing planned insertion would usually have awaited negative results before proceeding. As results take 4-7 days, emergency insertions must be carried out without Chlamydia status being known. This explains why all three positive results were in emergency insertions.

Some expulsions amongst the initial insertions have to be expected as the clinician is learning a new skill. As Liverpool has acted as a continuing training centre, many devices are inserted by clinicians relatively inexperienced in the technique of GyneFix insertion. This can account for expulsions within the first 3 months after insertion, but later expulsions are more difficult to explain. As a result of the finding that the expulsion rate is higher than quoted in the literature, a further follow-up study of the first 1000 insertions was commenced and will be reported separately. All IUD users are advised to check for the presence of the thread regularly. Expulsion should not be assumed if the device is not present on clinical and ultrasound examination; perforation should be excluded with a plain whole abdominal X-ray once pregnancy is excluded. In the Chinese randomised comparative study, the expulsion rate with the GyneFix is significantly lower than with the TCu380A. ${ }^{4}$ 
As follow-up is opportunistic and therefore incomplete, there may be removals and expulsions about which we are unaware. Removal and expulsion rates have been similar regardless of parity and whether insertion was planned or emergency, although small numbers and incomplete followup hamper statistical analysis.

Asking for any other comments at the end of the questionnaire proved a valuable insight. On the whole comments were encouraging and complimentary, both to the device itself and the experience of using the service. Some comments related to dissatisfaction with the increased menstrual flow, which may reflect unrealistic expectations about bleeding. As previously mentioned, this issue has been addressed.

\section{Conclusion}

The GyneFix intra-uterine device is well accepted in Liverpool in appropriately selected clients, and is an effective non-hormonal method for nulliparous as well as parous women. Most users are satisfied with the GyneFix and would recommend its use to a friend. In our experience, periods do become heavier with GyneFix, but most women find this acceptable. Expulsions may occur.
It is not possible from this study to determine whether the GyneFix performs better in terms of side effects than a framed IUD. The final results of the Chinese study might give more information, but only in the population studied. A randomised comparative trial including nulliparous women is needed.

\section{Statements on funding and competing interests}

Funding: Funding was provided by the National Co-ordinating Unit for Clinical Audit in Family Planning.

Competing interests: In lieu of payment for training other clinicians in the technique of GyneFix insertion, Abacus has received some devices free of charge from Contrel, the manufacturer.

References

Van Kets H, Vrijens M, Van Trappen Y, et al. The frameless GyneFix ${ }^{\circledR}$ intrauterine implant: a major improve Training in Human Reproduction, World Bank: IUD Research Group. The TCu 380A and the Frameless IUD "the FlexiGard": Interim three-year data from an international multicenter trial. Contraception 1995; 52: 77-83.

Rosenberg M, Foldesy R, Mishell D, et al. Performance of the TCu 380A and Cu-Fix IUDs in an international randomized trial. Contraception 1996; 53: 197-203.

Hu J and Wildemeersch D. Performance of the frameless GyneFix and the TCu $380 \mathrm{~A}$ IUDs in a three-year multicenter randomized comparative trial in parous women. Contraception

Chif Medical Officer's Expert Advisory Group on Chlamydia Trachomatis. Summary and conclusions of the CMO's Expert Advisory Group on Chlamydia Trachomatis. Departyent of Health, 1998.

6 Jones K, Darcy A, Webb A, et al. Screening, treating and contact tracing for asymptomatic Chlamydia infection in a young people's clinic (in Bootle) by Outreach Health Advisor is both acceptable and successful. European Journal of Contraception and Reproductive Health Care 2000; 5 (1): 53(a). 